\title{
Acute kidney injury due to systemic Loxoscelism: a cross- sectional study in Northeast Brazil
}

\author{
Polianna Lemos Moura Moreira Albuquerque ${ }^{[1],[2],}$ Louise Donadello Tessarolo ${ }^{[3]}$, \\ Fernanda Holanda Menezes ${ }^{[4]}$, Thayanne Barreto de Lima ${ }^{[4]}$, \\ José Hícaro Hellano Gonçalves Lima Paiva ${ }^{[5]}$, Geraldo Bezerra da Silva Júnior ${ }^{[6]}$, \\ Alice Maria Costa Martins ${ }^{[3]}$ and Elizabeth de Francesco Daher ${ }^{[1]}$
}

\author{
[1]. Programa de Pós-Graduação em Ciências Médicas, Faculdade de Medicina, Universidade Federal do Ceará, Fortaleza, CE, Brasil. \\ [2] Hospital Instituto Doutor Jose Frota, Prefeitura Municipal de Fortaleza, Fortaleza, CE, Brasil. \\ [3]. Programa de Pós-Graduação em Ciências Farmacêuticas, Faculdade de Farmácia, Universidade Federal do Ceará, Fortaleza, CE, Brasil. \\ [4]. Faculdade de Medicina, Universidade Federal do Ceará, Fortaleza, CE, Brasil. \\ [5]. Faculdade de Medicina, Universidade Estadual do Ceará, Fortaleza, CE, Brasil. \\ [6]. Programas de Pós-Graduação em Saúde Coletiva e Ciências Médicas, Faculdade de Medicina, \\ Universidade de Fortaleza, Fortaleza, CE, Brasil.
}

\begin{abstract}
Introduction: Loxoscelism is a clinical condition involving spiders of the genus Loxosceles. One of the most severe complications is acute kidney injury (AKI). This study aimed to investigate AKI and other complications associated with loxoscelism. Methods: We analyzed cases diagnosed with loxoscelism in an area where most accidents were caused by Loxosceles amazonica from January 2010 to December 2015. AKI was defined according to the KDIGO criteria. Results: Forty-five patients were recorded: 95.6\% presented characteristic necrotic skin lesions and 13.3\% AKI. Conclusions: Loxoscelism could cause kidney involvement which is uncommon and could lead to the death of these patients.
\end{abstract}

Keywords: Loxoscelism. AKI. Spider bites. Coagulation abnormalities.

Loxoscelism is a term used to cover envenomation involving spiders of the genus Loxosceles (family Sicariidae), known as recluse, fiddle-back, or brown spiders ${ }^{1}$.

In Brazil, seven species of these spiders have been reported, most of them in the South and South-East regions and three (Loxosceles intermedia, Loxosceles gaucho, and Loxosceles laeta) have been most implicated in human envenomations ${ }^{2}$. However, currently, Loxosceles amazonica has been described in some states in the North, Midwest and Northeast states, like Ceará ${ }^{3}$.

The diagnosis is made from epidemiological and clinical symptoms and findings ${ }^{2}$. We observed patients with the characteristic time-course of loxoscelism lesions and occasionally, non-specific systemic symptoms. Hence, the other clinical presentations were excluded.

Brown spider bites can have many different clinical repercussions, from small local inflammatory reactions to large necrotic skin degeneration and gravitational spread ${ }^{4}$, besides systemic effects.

Corresponding author: Dra. Polianna Lemos Moura Moreira Albuquerque e-mail: pollylemos78@gmail.com

Received 29 November 2017

Accepted 11 April 2018
The cutaneous form of loxoscelism occurs in most cases, and the systemic or viscerocutaneous form occurs less frequently ${ }^{2}$. Cutaneous loxoscelism manifests as mild pain and evolves into extensive skin necrosis and ulcerations. Systemic loxoscelism is characterized by fever, malaise, weakness, nausea and vomiting, hemolysis, hematuria, jaundice, thrombocytopenia, disseminated intravascular coagulation, and acute kidney injury (AKI), which is the main cause of death in loxoscelism ${ }^{2,5}$, although non-specific symptoms can occur in cutaneous loxoscelism.

AKI has been described in systemic loxoscelism in single case reports ${ }^{5-7}$. AKI induced by nephrotoxic agents such as spider venom, is a clinicopathologic entity morphologically characterized by the destruction of tubular epithelial cells and clinically by acute suppression of renal function ${ }^{4,8}$. Renal injury in loxoscelism can be attributed to pigmentary nephropathy due to hemoglobin or myoglobin, secondary to hemolysis or rhabdomyolysis ${ }^{9}$. Furthermore, some articles identified possible cellular and molecular mechanisms for nephrotoxicity due to Loxosceles spiders, indicating that Loxosceles venom is directly and potentially nephrotoxic ${ }^{4}$.

This study aimed to describe AKI and other complications associated with loxoscelism. This cross-sectional study covered envenomations due to brown spider bites from January 2010 
to December 2015, admitted to the Toxicological Assistance Center at Instituto Dr. José Frota Hospital, in Fortaleza City, Brazil. According to clinical presentation and anamnesis, the physician and pharmacologist toxicologist defined the diagnosis of loxoscelism, once the local lesion due to loxoscelism was extremely peculiar.

AKI is defined as any of the following according to KDIGO: (1) increase in serum creatinine level by $\geq 26.5 \mu \mathrm{mol} / 1(\geq 0.3 \mathrm{mg} /$ dl) within 48 hours or to 1.5 - 1.9 times the baseline value, or urine output $<0.5 \mathrm{ml} / \mathrm{kg} / \mathrm{h}$ for 6 - 12 hours; (2) increase in serum creatinine level to 2.0 to 2.9 times the baseline value, which is known or presumed to have occurred within the preceding 7 days, or urine output $<0.5 \mathrm{ml} / \mathrm{kg} / \mathrm{h}$ for $\geq 12$ hours; and (3) increase in serum creatinine level to 3.0 times the baseline value or to $\geq 4.0 \mathrm{mg} / \mathrm{dl}$, or urine output of $0.5 \mathrm{ml} / \mathrm{kg} / \mathrm{h}$ for 6 hours ${ }^{10}$.

The lowest creatinine level presented during hospitalization was considered as the baseline creatinine level. Two or more serum creatinine measurements were obtained from each patient studied during this period. Patients were classified according to the worst creatinine level.

Upon physical examination, signs and symptoms were evaluated and the following aspects were recorded: age, gender, Loxosceles genus (family Sicariidae), severity of condition, the time between the bite and administration of the antivenom, dose of antivenom, length of hospital stay, and hemorrhagic manifestations. The following laboratory parameters were evaluated: serum creatinine, urea, sodium, potassium, hematocrit, hemoglobin, leukocytes, platelets, prothrombin time, and partial activated thromboplastin time.

Patients were classified according to the severity of the brown spider bite using the Brazilian Ministry of Health criteria, based on local and systemic clinical manifestations established in specific tables for each case. The administration of specific antivenom was carried out according to those criteria.

A total of 45 cases were recorded. The mean age of the patients was 33.2 years [standard deviation (SD): 20.0], and there was a predominance of females $(\mathrm{n}=28 ; 62.2 \%)$. The majority of cases occurred in urban areas $(n=36 ; 80 \%)$. These cases were notified in the City of Fortaleza ( $\mathrm{n}=19 ; 42.2 \%)$, in Ceará, Northeast Brazil. The most frequently injured body sites were the lower limbs ( $\mathrm{n}=18 ; 40 \%)$, and the mean time between the bite and medical care was 144.54 hours (SD: 204.96). Clinical characteristics of the studied patients are described in Table 1.

In this study, most Loxosceles envenomations resulted in little more than a mild inflammatory reaction, so the patients did not undergo laboratory tests. These patients were evaluated at the Toxicological Assistance Center and by emergency department doctors, received medical prescriptions, and were discharged from the hospital.

Six patients presented with AKI associated with Loxosceles spiders. According to the KDIGO classification, three patients (numbers 2, 3 and 5) were classified as KDIGO 1, one was classified as KDIGO 2 (number 1), and two were classified as KDIGO 3 (numbers 4 and 6). Table 2 and Table 3 below show some of the results of these patients.

The six patients reported in Table 2 and Table 3 presented renal manifestations probably due to envenomation by Loxosceles during admission. The primary symptoms in all patients included fever, erythema, edema; one patient presented myalgia (number 6) and two presented jaundice, vomiting and nausea (numbers 1 and 4). Five patients presented platelet abnormalities, and developed changes in coagulation tests (numbers 1, 2, 4, 5, and 6) and three had anemia (numbers 1, 4 and 6).

Patients with AKI were hospitalized for medical treatment, requiring the administration of antivenom, antibiotics and steroids (numbers 1, 2, 4, and 5).

Two of them (numbers 2 and 5) had cardiorespiratory arrest, requiring mechanical ventilation. Hemodialysis was indicated in two cases, one of them (number 4) was discharged afterward, but the other died before this treatment could begin (number 6).

TABLE 1: Clinical characteristics of patients who experienced brown spider bites.

\begin{tabular}{lcc}
\hline Clinical characteristics & Number $(\mathbf{n}=\mathbf{4 5})$ & Percentage \\
\hline AKI & 6 & 13.3 \\
dialysis & 1 & 2.2 \\
Non-AKI & 39 & 86.7 \\
coagulation abnormalities & 8 & 17.8 \\
necrotic skin lesions & 43 & 95.6 \\
secondary infections complications & 24 & 53.3 \\
antibiotic administration & 27 & 60.0 \\
corticoids administration & 14 & 31.1 \\
antivenom administration & 35 & 77.8 \\
Dosage, mean (SD) & $4.8(2.5)$ & 2.44 \\
Death & 2 & \\
\hline
\end{tabular}

AKI: acute kidney injury; SD: standard deviation. 
TABLE 2: Laboratory parameters of six patients with acute kidney injury from envenomation due to Loxosceles.

\begin{tabular}{|c|c|c|c|c|c|c|}
\hline & Patient 1 & Patient 2 & Patient 3 & Patient 4 & Patient 5 & Patient 6 \\
\hline Laboratory parameters & $\begin{array}{c}75 \text { years } \\
\text { old, } \\
\text { female }\end{array}$ & $\begin{array}{c}38 \text { years } \\
\text { old, } \\
\text { female }\end{array}$ & $\begin{array}{c}72 \text { years } \\
\text { old, } \\
\text { male }\end{array}$ & $\begin{array}{c}42 \text { years } \\
\text { old, } \\
\text { male }\end{array}$ & $\begin{array}{c}60 \text { years } \\
\text { old, } \\
\text { female }\end{array}$ & $\begin{array}{c}47 \text { years } \\
\text { old, } \\
\text { male }\end{array}$ \\
\hline Initial Hb, g/dl & 12.6 & 13.4 & 13.6 & 7.6 & 15 & 6.7 \\
\hline Lowest $\mathrm{Hb}, \mathrm{g} / \mathrm{dl}$ & 8.1 & 12.9 & 11.9 & 7.6 & 12.8 & 6.1 \\
\hline Hematocrit, \% & 35.2 & 39.7 & 39.2 & 26.6 & 38 & 21 \\
\hline Platelets, $\mathrm{mm}^{3}$ & 140,000 & 525,000 & 224,000 & 37,000 & 95,000 & 524,000 \\
\hline Leukocytes, $\mathrm{mm}^{3}$ & 10,590 & 31,260 & 10,740 & 46,430 & 7,100 & 22,410 \\
\hline PT, seg & 12.7 & 17 & 11.6 & 14.5 & 14.9 & 15.6 \\
\hline aPTT, seg & 46.7 & 44.9 & 26 & 38.4 & 53.9 & 40.4 \\
\hline Baseline creatinine, $\mathrm{mg} / \mathrm{dl}$ & 0.4 & 0.6 & 0.6 & 1.3 & 1.9 & 1.8 \\
\hline Peak creatinine, mg/dl & 2.5 & 1.5 & 0.9 & 6.8 & 3.1 & 2.8 \\
\hline Initial urea, mg/dl & 68 & 48 & 33 & 21.3 & 126 & 103 \\
\hline Peak urea, mg/dl & 95 & 64 & 34 & 218 & 162 & 141 \\
\hline Initial sodium, mmol/l & 127 & 134 & 140 & 123 & 134 & 131 \\
\hline Peak sodium, mmol/l & 151 & 159 & ** & 133 & 134 & 147 \\
\hline Initial potassium, mEq/l & 3.29 & 4.9 & 3.8 & 5.2 & 5.4 & 5.68 \\
\hline Peak potassium, mEq/l & 4.25 & 4.9 & ** & 5.8 & 5.4 & 5.68 \\
\hline Initial magnesium, mg/dl & ** & 2.03 & 2.2 & 2.5 & ** & ** \\
\hline Peak magnesium, mg/dl & 2.04 & 2.27 & ** & 2.9 & ** & ** \\
\hline Initial calcium, mg/dl & 7.7 & 8.4 & 9.3 & 6.6 & ** & 9.7 \\
\hline Peak calcium, mg/dl & 8.5 & 9.5 & ** & 9.5 & ** & 9.7 \\
\hline Initial CPK, U/I & 268 & 187 & ** & 797 & ** & 17 \\
\hline Peak CPK, U/I & 268 & 651 & ** & 797 & ** & 406 \\
\hline Initial C-reactive protein, mg/l & 369 & 275 & ** & 142 & ** & 272 \\
\hline Peak C-reactive protein, mg/l & 369 & 275 & ** & 142 & ** & 308 \\
\hline Initial bilirubin total, mg/dl & 0.88 & ** & ** & 1.6 & ** & ** \\
\hline Peak bilirubin total, $\mathrm{mg} / \mathrm{dl}$ & 0.88 & $* *$ & ** & 2.3 & ** & ** \\
\hline Initial amylase, U/I & 18 & 678 & ** & 49 & ** & 114 \\
\hline Peak amylase, U/l & $* *$ & 678 & ** & 254 & ** & 114 \\
\hline Initial lipase, U/I & $* *$ & 1504 & ** & 45 & ** & 10 \\
\hline Peak lipase, U/I & $* *$ & 1504 & $* *$ & 317 & $* *$ & 16 \\
\hline Peak glucose, mg/dl & 142 & 539 & 120 & 102 & 128 & 114 \\
\hline
\end{tabular}

Hb: hemoglobin; g/dl: gram per deciliter; PT: prothrombin time; aPTT: activated partial thromboplastin time; CPK: creatine phosphokinase. ${ }^{*}$ nstitution reference values: hemoglobin 11.5-18g/dl; hematocrit 36-54\%; platelets 150,000-450,000mm; leukocytes 3,600-10,000mm³ PT 10-14s; aPTT 22-28s; creatinine 0.6-1.3mg/dl; urea 13-43mg/dl; sodium 135-146mmol/l; potassium 3.5-5.3mEq/l; magnesium 1.9-2.5mg/dl; calcium 8.5-10.5mg/dl; CPK <195U/L; C-reactive protein <6mg/l; total bilirubin 1.0; amylase 40-84U/l; lipase 13-60U/l. ** Not available

Only two patients had comorbidities; patient number 2 had diabetes and hypertension and number 4 had HIV and tuberculosis, discovered during hospitalization. In all patients, the bite progressed with local necrosis of varying severity, requiring, in four cases, surgical intervention by way of debridement and skin grafting. Among the six patients, two died (numbers 5 and 6), and four showed recovery from AKI and clinical improvement (numbers 1,2,3, and 4).

In the present study, the diagnosis of loxoscelism was based on epidemiological information and clinical evolution, similar to many studies described in the literature ${ }^{2}$. The cases were identified by typical characteristic skin lesions, necrotic lesions and systemic complications (AKI, coagulation abnormalities or liver abnormalities).

New geographic records of Loxosceles amazonica have been reported in Northern Brazil ${ }^{3}$, although there are no clinical studies regarding this species; thus, this is one of the first reports on this theme.

In this study, some patients developed secondary infections, requiring antibiotic therapy. The frequency of secondary 
TABLE 3: Clinical courses of six patients with acute kidney injury caused by Loxosceles envenomation in comparison with the literature.

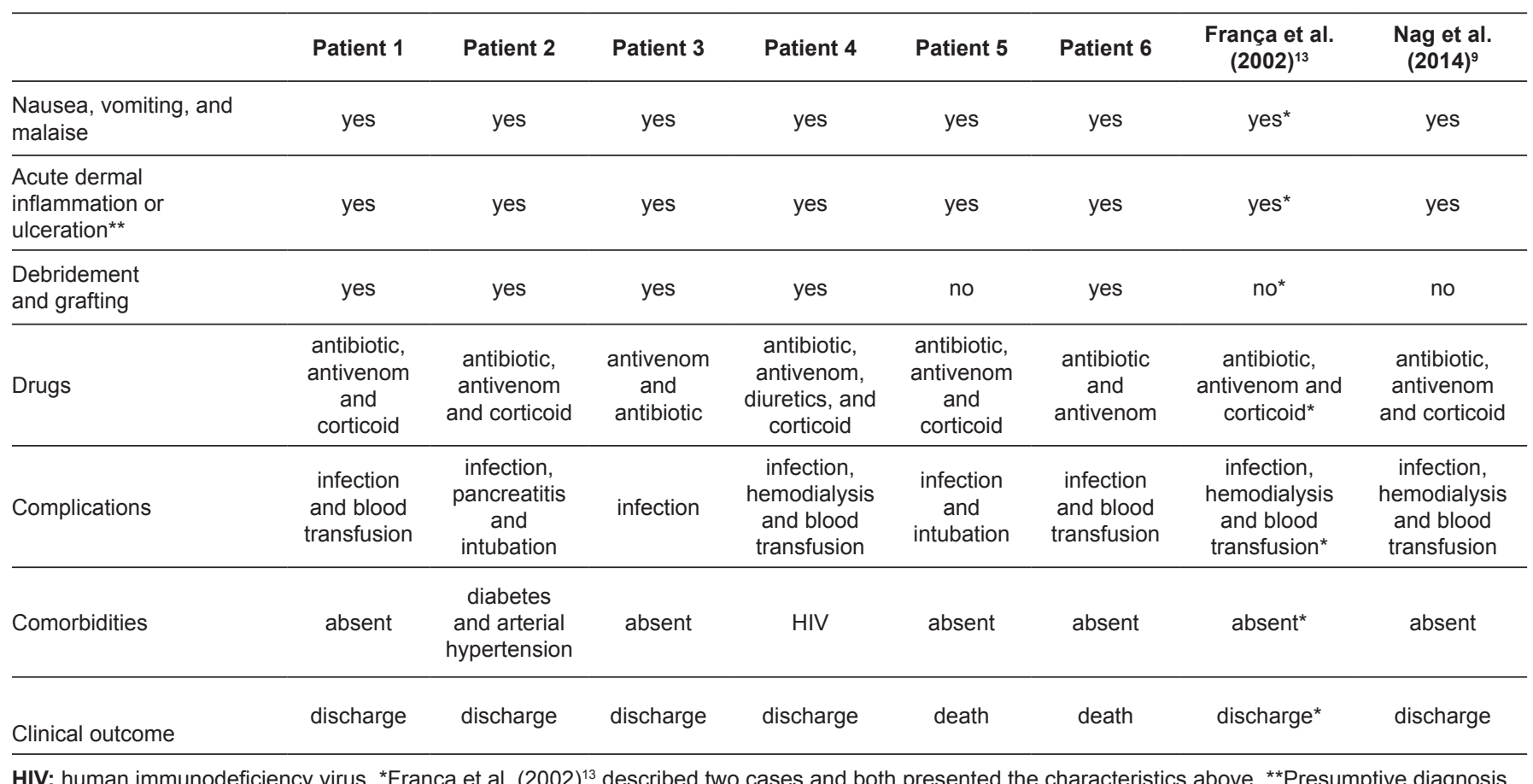

HIV: human immunodeficiency virus. *França et al. $(2002)^{13}$ described two cases and both presented the characteristics above. ${ }^{* *}$ Presumptive diagnosis.

infections could have been caused by some comorbidities of patients, such as malnutrition, alcohol consumption, diabetes, or acquired immunodeficiency syndrome (AIDS). Regardless of the severity of the injury caused by the envenomation, the development of secondary infection is unusual ${ }^{11}$.

AKI induced by loxoscelism has been described in in vitro and in vivo experimental studies ${ }^{4,5}$ and some cases have been reported $^{6}$. In this study, six patients were classified as having AKI according to the KDIGO classification.

Brown spider bites could have nephrotoxic effects ${ }^{4}$. The pathological effect of the venom on the kidney may reflect hematological disturbances, such as intravascular hemolysis, disseminated intravascular coagulation, the latter resembling atypical hemolytic uremic syndrome ${ }^{12}$.

Malaque et al. ${ }^{7}$ reported 81 patients diagnosed as cutaneous or cutaneous-hemolytic loxoscelism. AKI was described in two patients with massive hemolysis, but a positive correlation between creatinine and indirect bilirubin levels was not observed $^{7}$. Interestingly, in this present study, two of six patients with AKI presented indirect bilirubin levels higher than $0.7 \mathrm{mg} /$ $\mathrm{dl}$, which could indicate hemolysis. The other patients did not undergo tests for bilirubin.

A study conducted on mice exposed to L. intermedia venom ${ }^{4}$ observed a direct link between venom toxins and renal structures, suggesting Loxosceles toxins act as direct and potentially nephrotoxic agents. Due to their biochemical and physicochemical properties, venom toxins can act as planted antigens alongside the kidney structures, evoking nephrotoxicity ${ }^{4}$.
There are multiple causes of AKI in viscerocutaneous loxoscelism. Rhabdomyolysis as a result of intense tissue destruction was described in envenomations due to brown spiders ${ }^{13}$. The presence of pigmenturia in addition to hypovolemia is a very common cause of acute tubular necrosis ${ }^{13}$. In cases reported by França et al. ${ }^{13}$, patients presented myoglobinuria, hemoglobinuria, and bilirubinuria, which contributed to the development of AKI. In the present study, patients who developed AKI presented as first symptoms fever, erythema and generalized edema; however, only one patient presented myalgia while two presented jaundice. In the present study, four patients presented rhabdomyolysis, all of whom developed AKI. Two patients who developed AKI did not have their creatinine kinase levels measured.

In this study, only one patient, with AIDS and tuberculosis, underwent biopsy, which showed interstitial nephritis and acute tubular necrosis, similar to one of the cases described by França et al. ${ }^{13}$. The histological analysis of some organs from mice after envenomation with various doses of $L$. intermedia venom revealed remarkable alterations confined to the kidney. Acute tubular necrosis was seen in several nephrons, accompanied by deposition of eosinophilic material inside the proximal and distal renal tubules ${ }^{14}$. In another study, renal biopsies from L. intermedia venom-treated mice showed hyalinization and erythrocytes in the Bowman's space, glomerular collapse, tubular epithelial cell cytotoxicity and deposition of eosinophilic material within the tubular lumen. Ultrastructural studies showed glomerular epithelial and endothelial cell cytotoxicity, alterations of the basement membrane, and tubular epithelial cell degeneration ${ }^{4}$. 
The pathological effect of the venom on the kidney may produce hematological disturbances, such as intravascular hemolysis and disseminated intravascular coagulation, which are systemic manifestations of loxoscelism ${ }^{4}$. Hemolysis is well reported and may lead to hemoglobinemia, hemoglobinuria, renal failure ${ }^{9}$, and death ${ }^{15}$. In this study, three patients maintained acute hemolytic situations, presenting hemoglobin $<11 \mathrm{~g} / \mathrm{dl}$ and requiring blood transfusion. Abnormal bilirubin level was present in one case; five revealed platelet abnormalities and changes in coagulation tests.

The presence of systemic signals of loxoscelism such as intravascular hemolysis, intravascular coagulation, and renal failure can lead to the death of the victim; how this happened can be seen in the two cases described in the study.

Loxoscelism has been reported in urban areas in Northeast of Brazil. The main complications are necrotic cutaneous lesions, which could present secondary infections, coagulation abnormalities, and AKI.

The biggest challenge in the management of patients bitten by brown spiders is the clear diagnosis of the loxoscelism. The presumptive diagnosis is a limitation of this study. Otherwise, the spider species (Loxosceles amazonica) was not captured by the victims.

Further studies are essential to identify the causes of AKI due to brown spider bites, and in order to plan effective treatment to avoid death or severe complications.

\section{Ethical considerations}

The study protocol was reviewed and approved by the Brazilian Ethics and Research Council and the Ethics Committee of Instituto José Frota Hospital, in Fortaleza, Brazil (protocol number: CAAE: 41664214.5.0000.5052).

\section{Acknowledgements}

We are very grateful for the team of physicians, nurses, pharmacists, and technicians at the Center for Information and Toxicological Assistance of the Instituto Dr. José Frota Hospital, Fortaleza, Ceará, Brazil, for the exceptional care provided for these patients and for all others who seek care at this institution and for providing technical support for this study. We also thank the Brazilian Post-Graduation Coordination [Coordenação de Aperfeiçoamento de Pessoal de Nivel Superior (CAPES)] and National Research Council [Conselho Nacional de Desenvolvimento Cientifico e Tecnológico (CNPq)] for the financial support for our study group. Albuquerque PLMM received a grant from CAPES; Daher EF and Silva Jr GB received grants from CNPq.

\section{Conflict of interest}

The authors declare that there is no conflict of interest.

\section{REFERENCES}

1. Isbister GK, Fan HW. Spider bite. The Lancet. 2011;378(9808):203947.

2. Malaque CM, Castro-valença JE, Cardoso JL, França FO, Barbaro KC, Fan HW. Clinical and epidemiological features of definitive and presumed loxoscelism in São Paulo, Brazil. Rev Inst Med Trop. 2002;44(3):139-43.

3. Silveira AL. New geographic records of the brown spider Loxosceles amazonica Gertsch, 1967 (Araneae, Sicariidae) in Northeastern Brazil and its medical importance. Rev Med Minas Gerais. 2015;25(1):37-45.

4. Luciano MN, Da Silva PH, Chaim OM, dos Santos VL, Franco CR, Soares MF, et al. Experimental evidence for a direct cytotoxicity of Loxosceles intermedia (brown spider) venom in renal tissue. J Histochem Cytochem. 2004;52(4):455-67.

5. Lucato Jr RV, Abdulkader RC, Barbaro KC, Mendes GE, Castro I, Baptista MA, et al. Loxosceles gaucho venom-induced acute kidney injury - in vivo and in vitro studies. PLoS Negl Trop Dis. 2011;5(5):4-8.

6. De Souza AL, Loxosceles venom-induced cytokine activation, hemolysis, and acute kidney injury. Toxicon. 2008;51(1):151-56.

7. Malaque CMS, Santoro ML, Cardoso JLC, Conde MR, Novaes CTG, Risk JY, et al. Clinical picture and laboratorial evaluation in human loxoscelism. Toxicon. 2011;58(8):664-71.

8. Kusma J, Chaim Om, Wille AC, Ferrer VP, Sade YB, Donatti L, et al. Nephrotoxicity caused by brown spider venom phospholipase-D (dermonecrotic toxin) depends on catalytic activity. Biochimie. 2008; 90(11-12):1722-36.

9. Nag A, Datta J, Das A, Agarwal AK, Sinha D, Mondal S, et al. Acute kidney injury and dermonecrosis after Loxosceles reclusa envenomation. Indian J Nephrol. 2014;24(4):246-48.

10. Kidney disease: Improving global outcomes (KDIGO) acute kidney injury work group. KDIGO clinical practice guideline for acute kidney injury. Kidney Int Suppl. 2012;2(1):1-138.

11. Gremski LH, Trevisan-Silva D, Ferrer VP, Matsubara FH, Meissner $\mathrm{GO}$, Wille AC, et al. Recent advances in the understanding of brown spider venoms: From the biology of spiders to the molecular mechanisms of toxins. Toxicon. 2014;83:91-120.

12. Tambourgi DV, Magnoli FC, Berg CW, Morgan BP, Araujo PS, Alves EW, et al. Sphingomyelinases in the venom of the spider Loxosceles intermedia are responsible for both dermonecrosis and complement-dependent hemolysis. Biochem Biophys Res Commun. 1998;251(1):366-73.

13. França FO, Barbaro KC, Abdulkader RC. Rhabdomiolysis in presumed viscerous-cutaneous loxoscelism: reported of two cases. Trans R Soc Trop Med Hyg. 2002;96:287-90.

14. Tambourgi DV, Petricevich VL, Magnoli FC, Assaf SLMR, Jancar S, Silva WD. Endotoxemic like shock induced by Loxosceles spider venoms: pathological changes and putative cytokine mediators. Toxicon. 1998;36(2):391-403.

15. Mold JW, Thompson DM. Management of brown spider bite in primary care. J am Board Fam pract. 2004;17(5):347-52. 